\title{
Highly emissive fluorescent silica-based core/shell nanoparticles for efficient and stable luminescent solar concentrators
}

\author{
Francesca Corsini ${ }^{\text {a }}$, Elisavet Tatsi ${ }^{\text {a }}$, Alessia Colombo ${ }^{\text {b }}$, Claudia Dragonetti ${ }^{\mathrm{b}}$, Chiara \\ Botta $^{c}$, Stefano Turri ${ }^{\text {a }}$, Gianmarco Griffini *a \\ ${ }^{a}$ Department of Chemistry, Materials and Chemical Engineering “Giulio Natta”, Politecnico di Milano, Piazza Leonardo da Vinci 32, \\ 20133 Milano, Italy \\ ${ }^{b}$ Department of Chemistry, Università degli Studi di Milano, Via Camillo Golgi 19, 20133 Milano, Italy \\ "Institute of Sciences and Chemical Technologies “Giulio Natta” (SCITEC) of CNR, via Corti 12, 20133 Milano, Italy
}

\begin{abstract}
Luminescent solar concentrators (LSCs) represent a viable spectral conversion technology to maximize sunlight harvesting while promising to reduce the current gap for integration of solar cells into the built environment. In this work, novel highly emissive and photostable core-shell nanostructured luminescent species were synthesized and used in LSC devices. Such systems were obtained by entrapping Lumogen F Red 305 in silica-based shells by means of the sol-gel process using a combination of hydrophilic (tetraethyl orthosilicate) and hydrophobic (phenyl triethoxysilane) silica precursors. By tuning their relative proportions during the synthetic process, fine control over the characteristic dimensions and morphology of the obtained dye-doped core-shell nanoparticles could be achieved, leading to a maximum concentration of non-covalently entrapped fluorescent dye of $\sim 1$ wt.\%. Optical characterization showed that the newly synthetized nanoparticles displayed markedly improved photoluminescence quantum yield in solid state ( $95 \%)$ with respect to the non-encapsulated dye ( $2 \%$ ), as a result of an effective suppression of dye aggregation and fluorescence quenching phenomena. In addition, their excellent photostability under harsh UV light exposure was demonstrated, resulting from the protective action of the encapsulating hybrid silica-based shell, which can effectively limit photobleaching of the luminescent core. Their incorporation in thin-film LSCs led to a maximum internal photon efficiency as high as $\sim 29 \%$, thus providing evidence of their suitability as highly performing luminescent species with superior optical response and excellent photostability. Also, a maximum external photon efficiency as high as $2.12 \%$ was achieved. This work provides the first demonstration of silica-based encapsulation of Lumogen F Red 305 and of the application of silica-based core-shell nanoparticles in LSCs, thus paving the path to the development of a new class of highly efficient and stable nanostructured luminophores for the photovoltaic field.
\end{abstract}

Keywords: organic-dye-doped silica nanoparticles, core-shell nanoparticles, luminescent solar concentrators, photostability, photovoltaics. 


\section{Introduction}

The steady increase in energy demand and the simultaneous need of reducing carbon emissions caused by the overconsumption of fossil fuels for energy production represent some of the current drivers for continuous technological improvement in the field of renewable energy. In particular, the development of new efficient devices and the increase in performance of existing ones constitute topics of high priority for modern science and engineering. Among the many available energy sources, solar energy is by far the most readily accessible and exploited nowadays. Indeed, photovoltaic (PV) technology has evolved rapidly in the past few decades and now encompasses a large variety of materials and device structures, targeting both stationary and mobile applications [1],[2].

Within this framework, luminescent solar concentrators (LSCs) have been employed as a viable spectral conversion technology to maximize sunlight harvesting while promising to reduce the current gap for integration of solar cells into the built environment (BIPV)[3]. The basic idea behind the LSC concept is the replacement of a large solar-cell area with a transparent thin film or plate collector (the host matrix) containing luminescent species (the luminophores) that enable a large amount of incident photons to be absorbed, to be spectrally converted through a photoluminescence (usually fluorescence) process and to be redirected towards edge-mounted small-area PV cells, where the conversion of light to electricity can take place. Interestingly, the ability of luminophores to collect both direct and diffuse light strengthens the possibility to employ LSCs for BIPV applications and allows to improve the light-harvesting ability of conventional solar cells[4],[5].

In the past few years, research efforts in this field have largely focused on the elaboration of effective approaches to maximize the performance of LSC devices by focusing on the following three main aspects: the optimization of the device assembly, in the pursuit of more efficient architectures to boost photon harvesting, transport and conversion into usable electrical energy[6]; the development of highly performing luminescent species, to ensure broad light absorption and efficient photon emission[7-10]; the design of novel host matrices, to guarantee appropriate optical properties, sufficient chemical compatibility with the guest luminescent species and good processability for easy device fabrication[11],[12]. Similarly, a sufficiently long durability of LSC systems during operation also represents a key asset to prove the real commercial viability of this technology. In particular, two critical aspects directly affecting the lifetime of LSCs are the chemical and physical durability of the host matrix, and the photostability of the luminescent species, both addressed by designing intrinsically stable host materials [13-18] and luminophores [19-24].

In addition to more emerging classes of emitters such as inorganic phosphors and quantum dots [25-29], organic dyes have been traditionally the most widely employed in LSCs, likely due to their relatively straightforward synthetic accessibility and to the easy tunability of their optical properties by design. Among them, perylene-based systems (e.g., Lumogen F Red 305 by BASF, LR305 in the following) still represent the reference materials in the field, as they offer many attractive features including high absorption coefficients, quantum yields in solution approaching unity and good solubility in a wide range of polymer matrices. Nevertheless, these systems also present some important drawbacks that limit their optical performance in LSC devices, namely potential photo-instability resulting from the detrimental interaction with degradation products from the host matrix, strong aggregation-induced photoluminescence quenching, and potential photobleaching particularly when directly exposed to UV light [7],[30-32].

Taking inspiration from the biomedical field, and in particular from areas such as bioimaging, labelling, clinical diagnosis and drug delivery[33-37], a potential approach to overcome photobleaching and aggregation-induced photoluminescence quenching constraints is the development of core-shell nanoparticles (NPs), with organic luminescent molecules either physically or covalently entrapped in the core, and the protective shell being constituted by a layer of silica or by a polymeric material. In this context, the selection of the material used for the shell needs to be accurately weighted up, considering the chemical nature both of 
the entrapped luminescent dye and of the host matrix in which such NPs are meant to be incorporated. While polymeric shells may offer better chemical compatibility with the surrounding medium given that most LSCs are built using polymer-based host matrices (such as e.g. poly(methyl methacrylate) - PMMA), they can often provide little-to-no-protection to the dye molecules from external factors (e.g., solvents, air/oxygen), making these systems more prone to luminescence quenching and photobleaching under working conditions[38]. Therefore, NPs based on the use of silica as shell material can in principle be preferred in some applications, considering that silica exhibits high transmittance in the UV-visible spectrum, is photo-chemically inert[39], provides a chemically and mechanically stable vehicle for dye encapsulation, may serve as an effective protection layer for the luminophore thus improving its photostability, may prevent or limit dye aggregation, and is intrinsically nontoxic[40]. Moreover, from a synthetic point of view, silica is a very appealing material: the preparation of silica-based NPs usually requires relatively mild reaction conditions that can be adjusted to easily tune their size, while normally not involving complicated purification steps.

The most consolidated synthetic protocols for the formation of dye-silica core-shell NPs are the water-inoil reverse microemulsion method[41-44] and the Stöber method[45,46]. In both cases, only dyes exhibiting functional groups suitable for covalent attachment to the shell or carrying positive charges within their molecular structure can normally be considered for NP formation because covalent binding or electrostatic interactions are exploited to incorporate the organic molecule into the silica matrix. However, most of the organic dyes currently used as best performing luminophores in LSC systems (e.g., perylene-based) are highly hydrophobic or require intensive synthetic routes to equip their core structure with easily accessible groups suitable for covalent linking with the silica shell[47,48]. While attempts have recently been made to design new chemical pathways to enable the incorporation of hydrophobic organic dyes into silica NPs for improved photostability, brightness, and reduced molecular aggregation[34,39,49-52], only limited efforts have been addressed to the encapsulation of inert organic molecules via supramolecular interactions[40,53], mostly focusing on fluorescent systems of specific interest for the biological and biomedical fields[46,52,54]. On the contrary, no examples have been reported to date of the development of silica-based fluorescent NPs entrapping organic luminophores with suitable optical properties for PV and LSC applications, despite the potential advantages this approach can provide in terms of enhanced fluorescence efficiency, improved photostability and reduced molecular aggregation as compared with non-encapsulated dyes.

To bridge this gap, LR305-silica core-shell NPs (SiLRNPs) were synthesized in this work and employed as new nanostructured luminophore in thin-film LSC devices. These new SiLRNPs were obtained via the sol-gel process through a modified Stöber method[46] based on the hydrolysis and condensation of two silane reagents, namely phenyltriethoxysilane (PTES) and tetraethyl orthosilicate (TEOS), in the presence of LR305. The aromatic character of PTES enables supramolecular interactions with the dye molecules, allowing a stable LR305 suspension in the hydrophilic hydroalcoholic reaction medium and providing access to the formation of the encapsulating silica shell by sol-gel reaction with TEOS. The effect of TEOS:PTES relative proportions on dimension, morphology, chemical composition, dye encapsulation efficiency and chemical resistance of the synthetized fluorescent NPs was thoroughly investigated, and optimal process conditions for dye entrapment were highlighted. To provide evidence of the suitability of such novel SiLRNPs as highly performing luminophores, their optical properties were tested in thin-film LSCs and compared with control systems incorporating non-encapsulated LR305. This represents the first demonstration of silica-based encapsulation of LR305 and of the application of silica-based core-shell luminescent species in efficient and stable LSCs. 


\section{Material and Methods}

\subsection{Material}

Ethanol ( $\geq 99.8 \%$ ), ammonium hydroxide solution (28 vol.\%), tetraethyl orthosilicate (TEOS), phenyltriethoxysilane (PTES) and hexane (anhydrous, 95\%) were all obtained from Sigma-Aldrich. Lumogen ${ }^{\circledR}$ F Red 305 (LR305) was supplied by BASF, while Silgard 184, a two-component (component A:component $B=10: 1 \mathrm{w} / \mathrm{w}$ ) silicone elastomer resin used to prepare poly(dimethyl siloxane) - PDMS, was obtained from Dow. Index Matching Liquid 150 (IML150) was purchased from Norland. Monocrystalline silicon solar cells (mc-Si PV) were provided by IXYS (IXOLAR SolarBIT KXOB22-12X1F, active area $2.2 \times$ $0.6 \mathrm{~cm}^{2}-V_{\mathrm{OC}}=0.64 \pm 0.01 \mathrm{~V}, J_{\mathrm{SC}}=42.60 \pm 0.42 \mathrm{~mA} \mathrm{~cm}{ }^{-2}, F F=69.4 \pm 0.3 \%$, power conversion efficiency $=$ $18.69 \pm 0.23 \%)$.

\subsection{Synthesis of fluorescent silica nanoparticles}

The LR305-silica core-shell fluorescent NPs (SiLRNPs) were synthesized using the Stöber method with some modifications[46]. First, $0.4 \mathrm{~mL}$ of $10 \mathrm{mM}$ solution of LR305 in ethanol were transferred into a $25 \mathrm{~mL}$ round-bottom flask containing $12.6 \mathrm{~mL}$ of ethanol and were mixed with half of the total required volume of PTES ( $1^{\circ}$ PTES). Then, $0.20 \mathrm{~mL}$ of ammonium hydroxide were added to start the hydrolysis of PTES, and the solution was maintained under magnetic stirring at low speed for $24 \mathrm{~h}$ at room temperature. Subsequently, TEOS and the remaining volume of PTES ( $2^{\circ}$ PTES) dissolved in $5 \mathrm{~mL}$ of ethanol were added to the hydrolysed solution and further reacted with $0.10 \mathrm{~mL}$ of ammonium hydroxide solution. The reaction was carried out for an hour at $0{ }^{\circ} \mathrm{C}$ in the presence of continuous sonication (with an ultrasonic processor Sonics Materials $^{\mathrm{TM}}$ Vibra-Cell VCX130 at sonication amplitude 90\%), and then magnetically stirred for another $24 \mathrm{~h}$ at room temperature. The obtained NP suspension was centrifuged at $3300 \mathrm{rpm}$ and washed four times with a washing buffer solution of ethanol and DI water (25 vol.\%). The residue was left to dry completely at $80{ }^{\circ} \mathrm{C}$ in an oil bath followed by vacuum-oven treatment to completely remove water.

Five different batches of LR305-doped silica nanoparticles were prepared changing the volume ratio of silica precursors (TEOS:PTES ratio) and keeping constant the total amount of silica precursors (PTES+TEOS). Also, a control batch of non-loaded silica NPs (i.e., without LR305 and with TEOS:PTES = 1:1 v/v) was synthetized for direct comparisons. The exact quantities of precursors used for the synthesis of the different SiLRNP batches are reported in Table S1 in the Supporting Information.

\subsection{SiLRNP incorporation into LSC devices and coupling of mc-Si PV cells}

LSC devices in thin film configuration were prepared by incorporating the synthesized SiLRNPs at increasing concentrations into a PDMS-based host matrix obtained by thermally curing a two-component silicone elastomeric resin (Silgard 184). Control experiments were also conducted using bare LR305 as reference luminophore. Further details on the preparation of PDMS-based LSC devices are provided in a dedicated section (S.9) in the Supporting Information.

To achieve the LSC/PV assembly, the as-formed thin-film LSC systems were coupled to four mc-Si PV cells in series, so that two opposite edges of the glass substrate were connected to the photoactive area of two PV cells each. Bonding was achieved by means of IML150 ( 1.52 refractive index) that was dispensed on the active face of the PV cell. 


\subsection{Characterization techniques}

Scanning electron microscopy (SEM) and energy-dispersive X-ray spectroscopy (EDS) analysis were performed on gold sputtered SiLRNP samples using a Zeiss EVO 50 scanning electron microscope. Micrographs of core-shell NPs were acquired using a Philips CM200 transmission electron microscope (TEM) equipped with a field emission gun operated at $200 \mathrm{keV}$ accelerating voltage and starting from a $1 \mathrm{mg} / \mathrm{ml}$ solution of these particles dispersed in hexane. TEM and SEM images were analysed using ImageJ software to estimate the average NP diameter. More than 300 NPs were considered to obtain statistically relevant size distributions.

Thermogravimetric analysis (TGA) was performed using a TA Instruments Q500 thermogravimetric analyser. Measurements were carried out in air from 25 to $800{ }^{\circ} \mathrm{C}$, with a constant heating rate of $10{ }^{\circ} \mathrm{C} \mathrm{min}{ }^{-1}$. The amount of LR305 entrapped in SiLRNPs was determined by means of UV-Vis spectroscopy (Evolution 600 UV-vis spectrometer, Thermo Scientific) through leaking tests in two distinct organic solvents of different polarity (chloroform and ethanol). Based on the absorbance results, the kinetic of dye leakage from the silica capsules and the total amount of dye trapped in the NPs was estimated.

Luminescence quantum yield measurements (LQY) of SiLRNPs were performed with a C11347 Quantaurus-Absolute Photoluminescence Quantum Yield Spectrometer (Hamamatsu Photonics K.K), equipped with a $150 \mathrm{~W}$ Xenon lamp, an integrating sphere and a multichannel detector.

Accelerated aging tests were performed in combination with fluorescence spectroscopy to assess the photostability of the synthesized SiLRNPs and compared with that of control LR305 powder. In particular, 1 $\mathrm{mg}$ of each of the considered solid powder (LR305 or SiLRNPs) was sandwiched between two quartz coverslips. Then, the samples were exposed to continuous UV-A light (radiative power hitting the powder was $54.6 \mathrm{~mW} \mathrm{~cm}{ }^{-2}$ ) in air at a temperature of $50^{\circ} \mathrm{C}$ and their emission spectra were collected every $30 \mathrm{~min}$. A UV polymerization apparatus (POLIMER 400W, by Helios Quartz) was used as light source for the UV-A irradiation experiments, equipped with high pressure mercury lamps with emission window in the 315-400 nm wavelength range. Accelerated aging experiments were repeated on SiLRNP-PDMS LSCs and LR305-PDMS LSCs to evaluate the photostability of the final devices under the same testing conditions. Time-resolved fluorescence measurements by time-correlated single photon counting (TCSPC) technique were carried to verify the effective encapsulation of the organic dye in the core of silica NPs and they were obtained with a NanoLog composed by a iH320 spectrograph equipped with a PPD-850 single photon detector module and a DD-405L DeltaDiode Laser. The decays were analysed with the instrument Software DAS6. Steady-state fluorescence spectroscopy measurements in front-face configuration on LR305 solid powders, SiLRNPs and LSC thin-film devices were carried out in air at room temperature using a Jasco FP-6600 Spectrofluorometer. The photoluminescence emission spectra were recorded from 550 to $800 \mathrm{~nm}$ wavelength range exciting at 445 $\mathrm{nm}$, while the excitation spectra were collected at the emission wavelength of $615 \mathrm{~nm}$ by scanning the excitation wavelength from 280 to $600 \mathrm{~nm}$.

Diffuse reflectance spectra of undoped-PDMS LSC and SiNP-PDMS LSC were collected with a Jasco V570 UV-Vis-NIR instrument combined with an integrating sphere. Absorbance measurements of LSC systems were carried out with an ILT950 spectroradiometer and SpectraLight III software. Illumination of LSC devices and LSC/PV assembly was performed using a calibrated Sun 2000 solar simulator (Abet Technologies) equipped with an AM 1.5G filter and imposing solar irradiation at 1 sun $\left(100 \mathrm{~mW} \mathrm{~cm}^{-2}\right)$. The edge emission irradiance spectrum emitted from each of the four edges of the LSC in the presence of an absorbing black background was collected by the spectroradiometer equipped with a cosine corrector positioned at the center of the edge, while the other edges were covered with black tape. The parameters used to characterize the optical performance of LSCs were the internal photon efficiency $\eta_{\text {int }}$ and the external photon efficiency $\eta_{\mathrm{ext}}$ (Equation S4 and Equation S5 in Supporting Information). 
Current-voltage characterization of the illuminated LSC/PV assembly was performed using a Keithley 2612B multimeter to carry out the voltage scans and acquire the output photogenerated current. PV tests were repeated with two different experimental setups:

- fixing an absorbing black background in contact with the LSC back surface to avoid photocurrent overestimation due to photon double-pass effects;

- placing a white diffuser (Edmund Optics, \#34-480) in contact with the rear side of the LSC to allow back scattering of transmitted photons.

In both cases, a black mask placed on the front face of the LSC system was used to avoid direct illumination of the PV cells, thus leading to undesirable photocurrent overestimation. Also, no reflective element was employed at the free edges of the waveguide. These measurements enabled to determine the device efficiency $\eta_{d e v}$ defined as the electrical power effectively extracted from the PV cells ( $\left(P^{\text {out }}{ }_{e l}\right)$ relative to the optical input power hitting the top surface of the LSC $\left(P^{\text {in }}{ }_{\text {opt }}\right)$ :

$\eta_{\text {dev }}=\frac{P_{e l}^{\text {out }}}{P_{o p t}^{\text {in }}}=\frac{F F \cdot I_{S C} \cdot V_{O C}}{P_{o p t}^{\text {in }} \cdot A_{L S C}}$

where $F F, I_{S C}$ and $V_{O C}$ are the fill factor, the short-circuit current and the open-circuit voltage of the edgecoupled PV cells, respectively, $P^{i n}{ }_{\text {opt }}$ is the incident solar power density (in $\mathrm{mW} \mathrm{cm}{ }^{-2}$ ) and $A_{\mathrm{LSC}}$ is the front illuminated area of the LSC device $\left(\right.$ in $\left.\mathrm{cm}^{2}\right)$.

\section{Results and discussion}

\subsection{SiLRNPs synthesis and characterization}

SiLRNPs were synthesized through a two-step hydrolytic condensation process[46], where the aromatic silane precursor PTES was first allowed to undergo self-condensation reaction around LR305 molecules, followed by the growth of a silica shell surrounding the entrapped dyes via condensation reaction after the addition of a mixture of TEOS and PTES. Because LR305 is a neutral species and does not possess functional groups readily available for covalent bonding with the silica layer, this two-step approach enables supramolecular physical entrapment of the dye molecule inside a silica shell by exploiting hydrophobic and $\pi$ $\pi$ stacking interactions between the aromatic groups of PTES and LR305 (see Figure 1). As a result, the formation of a core-shell NP morphology with a diffuse interphase is expected.

In order to validate this assumption, SEM-EDS analysis was carried out on SiLRNPs (TEOS:PTES = 1:1 $\mathrm{vol} / \mathrm{vol}$ ) as well as on pristine LR305 dye powder and blank silica NPs, obtained in the same conditions as SiLRNPs but in the absence of LR305 (SiNPs). All EDS spectra and the corresponding average elemental percent abundance are reported in Figure S1 and discussed in depth in the Supporting Information. Briefly, this preliminary evaluation revealed that a core-shell structure can be assumed for such SiLRNPs, where LR305 molecules are entrapped as core within a surrounding silica shell. The present findings were corroborated by the results obtained from time-resolved fluorescence measurements, from which the average fluorescence lifetime values and the nonradiative decay rate constants were evaluated. Figure 2 compares time resolved fluorescence decay curves for LR305 and SiLRNPs in the solid state. The corresponding average fluorescence lifetime values were found to be equal to 0.31 ns and $7.73 \mathrm{~ns}$, respectively. The pronounced increase in fluorescence lifetime of SiLRNPs proves the encapsulation of LR305 into the core of silica NPs[55-57]. Indeed, the longer fluorescence lifetime of SiLRNPs is strongly coupled to the decrease in fluorescence quenching, given by the reduced level of aggregation of the dye upon encapsulation, and to the 
restricted conformational mobility of LR305 dye in the rigid silica environment. This latter aspect was confirmed by the reduced nonradiative rate value for SiLRNPs with respect to bare LR305, being the nonradiative rate sensitive to the architecture of the luminophore[58] (see Supporting Information for further details). Interestingly, all SiLRNPs produced varying TEOS:PTES proportions were found to exhibit similar fluorescence lifetimes, highlighting excellent reproducibility of the synthetic protocol and confirming a similar morphology for all systems.

Size, shape and morphology of SiLRNPs were investigated by means of TEM and SEM analyses (SEM images and size distribution profiles of every SiLRNPs batch are reported in Figure S3 and Figure S4 in the Supporting Information). As shown in Figure 3, where representative micrographs of SiLRNPs are reported, round nanostructures were obtained in all cases, with average particle sizes ranging from $20 \mathrm{~nm}$ to $200 \mathrm{~nm}$ and depending on the relative amounts of the sol-gel monomer precursors. In particular, the lower the TEOS:PTES ratio, the bigger the particle diameter (see Figure 3 on the lower left). This progressive increase in dimensions with the increased amount of aromatic precursor in the initial sol-gel formulation may be attributed to stronger hydrophobic, $\pi-\pi$ stacking interactions taking place as the TEOS:PTES ratio decreases. Additionally, this trend may also be associated to a change in the rates of the hydrolysis and condensation steps as the volume ratio between the sol-gel precursors is varied, as also reported in previous studies on similar sol-gel systems[59,60]. Indeed, when the single PTES precursor is employed for NP formation (a situation corresponding to the first step of the hydrolysis/condensation process reported here for SiLRNP production), the hydrolysis rate of PTES was found to decrease for higher PTES concentrations, accompanied by a faster condensation process. This trend was shown to lead to the formation of bigger NPs. As opposed to this, when a mixture of TEOS and PTES is used (a situation corresponding to the second step of the hydrolysis/condensation process reported here for SiLRNP production where both precursors simultaneously react), the increasing concentration of PTES was found to speed up the hydrolysis process of TEOS, while slowing down its condensation rate and simultaneously increasing the condensation rate of the aromatic silane precursor. These differences in the sol-gel process kinetics may first lead to the condensation of PTES, and then of TEOS, thus promoting the formation of NPs with a sharper core/shell interface. Similarly, it may yield an incomplete condensation of TEOS during the synthetic process. As a result, bigger NPs are found for lower TEOS:PTES ratios. It is interesting to observed that all SiLRNP systems exhibited a clearly visible core-shell morphology (with shell thickness $25-35 \%$ particle diameter as illustrated in Figure S2 in Supporting Information), further supporting what previously inferred from EDS and time-resolved fluorescence lifetime analyses and providing evidence of the robustness of the proposed synthetic protocol to achieve effective entrapment of the dye molecule within the outer silica layer. The optimization of the NP structure (e.g., synthetic control of shell thickness and core size) is currently underway and will be the subject of a separate study.

TGA measurements were conducted on all SiLRNP systems to further investigate the chemical composition of the obtained fluorescent nanostructures and to quantify the relative amounts of encapsulated dye as a function of sol-gel formulation. For comparative purposes, TGA was also performed on LR305 and blank SiNPs. As expected and in accordance with theorical predictions, a greater TEOS:PTES volume ratio in the sol-gel formulation resulted in a reduced amount of aromatic organic phase in the hybrid silica shell (see Figure S7.a, Figure S8 and Table S3 in the Supporting Information). Moreover, the encapsulation efficiency was found to increase as a result of stronger $\pi-\pi$ stacking interactions between the perylene-based molecular structure of LR305 and the aromatic ring in PTES (Table S4 and Figure S9 in the Supporting Information).

To further confirm these results and to accurately estimate the amount of entrapped-dye in the core-shell nanostructures, leakage tests were undertaken on SiLRNPs with increasing TEOS:PTES ratio in two distinct organic solvents of different polarity (i.e., chloroform - $\mathrm{CHCl}_{3}$ and ethanol - EtOH) and the absorbance of the solution of the extracted dye in the solvent was measured at regular time intervals through UV-vis 
spectroscopy. In particular, dye release tests with $\mathrm{CHCl}_{3}$ were conducted to estimate the maximum mass (wt.\%) of LR305 entrapped in the obtained SiLRNPs, while tests performed in EtOH were used to evaluate the kinetics of the release process. The successful entrapment of the dye molecules in the silica-based NPs could be demonstrated in both cases, as a result of the hydrophobic and $\pi-\pi$ stacking interactions between the extended aromatic structure of LR305 and the aromatic ring in PTES, with amounts of entrapped dye found to lie in the 0.57 - 1 wt.\% range (Figure S10 and related discussion in the Supporting Information).

\subsection{Photophysical characterization of SiLRNPs}

The photophysical properties of SiLRNPs with different TEOS:PTES ratios were investigated and compared to those of pure LR305 to determine the effects of encapsulation on the fluorophore luminescence quantum yield (LQY), photoluminescence emission/excitation spectra and photostability. The LQY of SiLRNPs in powder state was measured to be $95 \pm 0.2 \%$, a remarkably higher value with respect to pure nonencapsulated LR305 in the solid state (1.9\%), where LR305 is known to undergo fluorescence quenching due to the formation of non-luminescent aggregates (see Section S.7 in the Supporting Information). This result provides a first indication of the viability of the proposed strategy to obtain highly luminescent nanostructures and suggests a significant reduction of dye aggregation phenomena within the NPs.

Steady-state photoluminescence spectra for all five batches of SiLRNPs in the solid state (powder form) are reported in Figure 4(a), while excitation and emission spectra of pure LR305 dye and SiLRNPs-1:1 (taken as reference) are compared in Figure 4(b). The photoluminescence spectra of SiLRNPs with different TEOS:PTES proportions were found to exhibit the same emission profile, but with different maximum intensities and emission peak positions. In particular, by reducing the TEOS:PTES volume ratio, a progressive redshift in the emission wavelength was detected (with the exception of the NP batch prepared with TEOS:PTES = 1:1). Conversely, the maximum fluorescence intensity was found to show an opposite trend with respect to the amount of encapsulated LR305, being maximum for TEOS:PTES = 1:1. These results may be attributed to the effect of some fluorescence quenching starting to take place when surpassing a critical amount of dye molecules in the NPs, which is also compatible with the simultaneous redshift in the emission wavelength[61-64]. Comparing the photoluminescence spectra of bare LR305 dye and SiLRNPs, no significant differences were observed in their profiles, likely suggesting that the nature of the excitation and emission processes for pure dye and for dye entrapped in silica-based NPs is analogous and that any optical interaction between dye molecules and the silica shell can be excluded. Interestingly, it was also found that the peaks in excitation and emission spectra of SiLRNPs exhibited a 15-30 nm blue shift (depending on the considered batch) when compared with free LR305, while their fluorescence intensity was remarkably higher. This hypsochromic shift could be attributed to the polarizability of the environment and/or to the restricted motion of molecules when LR305 is caged inside silica shell of the nanoparticles[37,38,65]. In particular, an eight-times more intense photoluminescence signal was recorded for SiLRNPs than for LR305 in the presence of the same amount of analysed material, in spite of the significantly lower net mass of luminophore (0.5-1 wt.\% of LR305 dye molecules in $1 \mathrm{mg}$ of SiLRNPs, as evidenced by extraction tests). These results clearly demonstrate that the nanostructured architecture of the obtained SiLRNPs significantly prevents dye aggregation and greatly limits dye fluorescence quenching, likely due to the fact that upon entrapment the luminophore is present as molecularly dispersed species in the hybrid silica-based NP core.

The stability of SiLRNPs against photobleaching was also investigated, being this aspect of key importance when the target application (i.e., LSC devices) is considered. To examine the effect of encapsulation on LR305 photostability, accelerated aging tests on all SiLRNP batches were carried out and compared with pure LR305. The samples, in solid powder form, were exposed in air to UV-A light for a maximum duration of $2 \mathrm{~h}$ 
and fluorescence spectra were taken every 30 minutes. As depicted in Figure 5, where the normalized evolution of the fluorescence emission peak intensity for both SiLRNPs (TEOS:PTES = 1:1) and LR305 is reported as a function of UV exposure time, in the case of pure LR305 a rapid and sharp emission decay was observed ( $\sim 53 \%$ loss after $2 \mathrm{~h}$ ) associated with the direct photobleaching of the fluorophore[66], in agreement with previous literature reports on the same system[31,67]. Conversely, a significantly slower and less pronounced reduction in photoluminescent intensity was detected for SiLRNPs under the same illumination conditions. In agreement with previous studies on different luminescent molecules[46,53], these results provide a direct demonstration of the enhanced photostability of our new luminescent nanostructures, where the organic-inorganic hybrid silica-based shell serves as protective layer for the entrapped LR305 luminophore thus effectively limiting its photodegradation, even in such highly aggressive exposure conditions.

\subsection{Optical characterization of LSCs incorporating SiLRNPs}

Considering the favourable chemical, physical and optical characteristics of SiLRNPs discussed above, their suitability as luminophores for LSC devices was investigated. A commercially-available organicinorganic hybrid host matrix (PDMS) was selected as the transparent waveguide due to its excellent photostability and suitable chemical-physical and optical properties, which make it a convenient reference platform to evaluate the properties of the new nanostructured luminophores in LSCs[11,68-70]. LSC devices in thin-film configuration obtained by solvent-free dispersion of SiLRNPs in PDMS host matrix (SiLRNPPDMS LSCs) were fabricated and their optical response was examined at increasing SiLRNP loading. For comparison, LSC systems based on pure LR305 dye (LR305-PDMS LSCs) were also prepared as reference, incorporating equivalent concentrations of luminophore species such that the optical density of LR305-PDMS LSCs was corresponding to that of SiLRNP-PDMS LSCs. The effect of luminophore concentration on the optical properties of PDMS-based LSCs was explored by means of both UV-vis absorption and fluorescence emission spectroscopy.

As can be noticed comparing Figure 6(a) and Figure 6(c), the characteristic absorption features of LR305 could be clearly distinguished in both PDMS-based LSC systems even if a non-negligible scattering-like background typical for silica NPs was noticed in SiLRNP-based systems (for a deeper account on the role of Rayleigh scattering on the optical performance of devices, diffuse reflectance and edge emission measurements are discussed in detail in the Supporting Information).

From the comparison of absorption spectra of LR305- and SiLRNPs-PDMS LSC devices, a greater affinity of the nanostructured luminophore vs. pristine LR305 to the host matrix was observed. This was conclusively proven by assessing the emission spectra of both PDMS-based LSC systems (Figure 6(b) and Figure 6(d)). In agreement with previous studies[71], beyond a very low LR305 threshold concentration (i.e., 0.1 wt.\%), a remarkable decrease in the fluorescence intensity was observed in LR305-PDMS systems, despite the increase in absorbance. Moreover, a bathochromic shift of the emission peak was observed by increasing LR305 concentration, accompanied by a noticeable spectral distortion of the emission profile. These trends are indicative of the presence of non-negligible dissipative processes, which cause a loss of part of the absorbed photons via non-radiative pathways, both through reabsorption of emitted photons due to the small Stokes shift of the dye and due to the formation of molecular aggregates through $\pi$ - $\pi$ stacking interactions[68,72]. Conversely, only a negligible bathochromic shift of the emission peak and no spectral distortion was observed for SiLRNPs within this concentration range in PDMS, indicating the reduced tendency of these nanostructured luminophores to form non-luminescent aggregates.

Finally, as illustrated in Figure 7, when comparing the maximum emission intensities of LR305- and SiLRNP-PDMS LSCs as a function of LR305 equivalent concentration, the nanostructured systems were found to significantly outperform the reference counterpart. The superior fluorescence intensity of SiLRNP- 
PDMS LSCs with respect to LR305-PDMS LSCs further confirms the potential of such LR305-containing nanostructured fluorescent systems as efficient luminophores for LSC applications.

On the basis of the promising results reported so far, the optical performance of LR305- and SiLRNPsPDMS LSC systems were evaluated and compared. One of the key parameters to characterize the performance of LSCs is the external photon efficiency $\eta_{\text {ext }}$, defined as the ratio of the total output photon flux measured at the four edges of the LSC $\left(\mathrm{N}_{\text {ph-out }}\right)$ with respect to the incident photon flux $\left(\mathrm{N}_{\text {ph-in }}\right)$ (Equation S5 in Supporting Information). As regards LR305-PDMS LSCs, $\eta_{\text {ext }}$ was found to increase with concentration, reaching a maximum $\eta_{\text {ext }}=0.93 \%$ at 0.1 wt. $\%$ (Figure $8(\mathrm{a})$ ). At higher concentrations, $\eta_{\text {ext }}$ was found to decrease in line with the optical loss pathways occurring at high doping levels described above (numerical results and optical power output spectra are reported in Table S5 and Figure S19 in the Supporting Information). Conversely, a steady increase in $\eta_{\text {ext }}$ with concentration was detected in SiLRNP-PDMS LSC systems with a value of $\eta_{\text {ext }}=$ 2.12\% for LR305 equivalent concentration of 0.5 wt.\%. Moreover, for each and every LR305 equivalent concentration, a higher $\eta_{\text {ext }}$ for SiLRNP-PDMS LSC devices was detected compared to that found in the corresponding LR305-PDMS LSC systems.

To better assess the contribution of the luminophore to the photon transport process within the waveguide, the internal photon efficiency ( $\eta_{\text {int }}$ ) was also calculated according to Equation S4 in Supporting Information). This figure of merit is related to the probability that a photon reaches the edge of the waveguide after being absorbed and emitted by the luminescent center; therefore, it gives an indication of how well the individual dyes and the lightguide process the absorbed light[73-75]. As can be seen from Figure 8(b), LR305-PDMS LSCs exhibit a maximum $\eta_{\text {int }}=14.03 \%$ for highly dilute systems (LR305 0.001 wt.\%), followed by a sharp decrease for progressively higher luminophore loadings. Instead, in SiLRNP-PDMS LSC devices, a maximum $\eta_{\text {int }}=28.74 \%$ was found for SiLRNPs concentration of 0.1 wt.\% (i.e., LR305 0.001 wt.\%), while in the 3-50 wt.\% SiLRNPs concentration range (LR305 equivalent concentration of 0.03-0.25 wt.\%) a steady $\eta_{\text {int }}$ value was detected $(\sim 13 \%)$. The higher $\eta_{\text {int }}$ found in SiLRNP-PDMS LSCs in comparison with the corresponding LR305-PDMS systems at the same LR305 equivalent concentration can be understood in terms of higher LQY of SiLRNP luminophore species and of reduced quenching losses (nonradiative relaxation pathways). These outcomes are comparable with that of colloidal fluorescent particles recently proposed in literature[76-82], further proving the practical viability of our nanostructured luminophore.

In addition to their superior optical properties, another important benefit of dye encapsulation in silica shell is the significant improvement of long-term photostability of the luminophores when embedded in the final system (i.e., PDMS matrix). As shown in Section S.14 in the Supporting Information, SiLRNP-PDMS LSCs exhibited excellent photostability when subjected to long-term ( $>250 \mathrm{~h}$ ) accelerated aging tests as opposed to LR305-PDMS LSCs, further confirming the factual potential of SiLRNPs as luminophore species for photostable and high efficiency LSCs.

\subsection{PDMS-based LSCs/PV Assembly}

Finally, to assess the performance of the LSC-PV assembly, photocurrent measurements were accomplished by using mc-Si PV cells mounted to two opposite edges of the concentrator plate, as described in the Experimental section. The overall (four edges) $\eta_{\text {dev }}$ of LR305- and SiLRNPs-PDMS LSCs for the various luminophore concentrations (wt.\%) was evaluated both in the presence of an absorbing black background ( $\left.\eta_{\text {dev,dark background }}\right)$ and using a white scatterer ( $\left.\eta_{\text {dev, white scatterer }}\right)$ in contact with the rear side of the LSC. The efficiency values are reported in Table 1 (plots are shown in Figure S22 in the Supporting Information). 
In line with the previous results, the PV performance of LR305-PDMS LSC devices was found to be optimized for a LR305 concentration of 0.1 wt.\%. In the presence of a black absorbing background, a maximum IsC of $3.61 \mathrm{~mA}$ and a maximum output power of $0.86 \mathrm{~mW}$ were achieved, leading to a maximum $\eta_{\text {dev,dark background }}$ of $0.24 \%$. The lower overall device efficiencies of LR305-PDMS LSCs with respect to previously published LR305-based thin-film LSCs tested in analogous conditions (namely, in the presence of a black absorbing background and of a black mask in front of the LSC system, without employing any reflective element at the free edges of the waveguide) $[15,83$ ] could be attributed to the poor luminophore solubility of LR305 in PDMS host matrix. As documented in previous studies[68,71,72], dye aggregation issues are responsible for generating significant luminescence quenching, which may limit thin-film device performance at higher dye concentrations, resulting in LR305-PDMS LSCs being optimized for lower optical densities. It is also interesting to note that a significant impact of the experimental configuration on the performance of PDMS-based LSC devices was observed. In accordance with previous studies[75,84-86], the overall device efficiency in the presence of a white scatterer at the rear side of an LSC resulted in a $35 \%$ improvement. Indeed, back scattering and reflection of otherwise lost unabsorbed light contribute significantly to enhance LSC performance. On the contrary, in the same experimental conditions, notably higher efficiency values were detected for SiLRNP-PDMS LSC systems relative to LR305-PDMS LSC with comparable equivalent LR305 concentration. Indeed, SiLRNPs-PDMS LSC containing $10 \mathrm{wt} \%$ of SiLRNPs (equivalent LR305 concentration $=0.1 \mathrm{wt} . \%$ ) displayed a maximum IsC of $4.96 \mathrm{~mA}$, a maximum output power of 1.43 $\mathrm{mW}$, and $\eta_{\text {dev,dark background }}$ of $0.49 \%$. Moreover, increasing SiLRNPs concentration, a further enhancement in

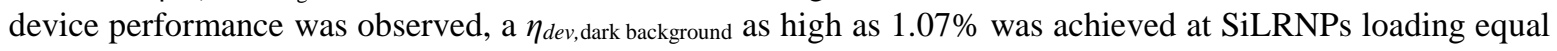
to 50 wt.\%, which account for a 460\% enhancement compared with the pure LR305 based LSC devices with the same luminophore equivalent concentration. The present results, that are in line with most recent literature[77,82,87], could be ascribed to the reduced tendency of these nanostructured luminophores to aggregation and to the improved LQY.

\section{Conclusions}

In conclusion, novel nanostructured luminescent species based on hybrid core-shell fluorescent NPs featured by enhanced LQY and photostability were synthetized, characterized and used as highly emissive fluorophores in thin-film LSC devices. Such systems were obtained for the first time by entrapping LR305 in silica-based shells by means of the sol-gel process using a combination of hydrophilic (TEOS) and hydrophobic (PTES) silica precursors. By tuning the relative proportions of TEOS and PTES during the synthetic process, fine control over the characteristic dimensions of the obtained NPs and the amount of noncovalently entrapped organic dye molecule could be achieved. As opposed to non-encapsulated LR305, the obtained fluorescent silica-based NPs were found to be highly emissive (LQY = 95\%) even in the solid state, as a result of an effective suppression of dye aggregation and fluorescence quenching phenomena. In addition, they demonstrated excellent photostability under harsh UV light exposure thanks to the protective action of the encapsulating hybrid silica-based shell, which can effectively limit photobleaching of the luminescent core. In the light of their notable photophysical properties, SiLRNPs were demonstrated as highly promising luminophore candidates in thin-film LSC devices with superior optical response with respect to systems based on non-encapsulated LR305.

This work provides the first demonstration of silica-based encapsulation of LR305 and of the application of silica-based core-shell luminescent species for LSCs, thus opening the path to the development of a new class of highly efficient and stable nanostructured luminophores for the PV field. 


\section{Conflicts of interest}

The authors declare that they have no known competing financial interests or personal relationships that could have appeared to influence the work reported in this paper.

\section{Acknowledgements}

Authors greatly acknowledge Gigliola Clerici for her kind support with thermal analysis.

\section{Vitae}

Gianmarco Griffini is currently Senior Assistant Professor (with tenure track) at Politecnico di Milano (Italy), where he received his MSc degree in Chemical Engineering in 2005 and his PhD degree in Materials Engineering in 2012. He has held visiting positions at University College London (UK), University of California at Berkeley (USA), and Universidad de Castilla-La Mancha (Spain). His current research interests are mainly focused on: materials and devices for solar light harvesting, managing and conversion; polymers for energy storage applications; high-performance stimuli-responsive polymeric materials for advanced manufacturing technologies; biodegradable and bio-derived polymers.

Francesca Corsini completed her MSc degree in Materials Engineering and Nanotechnology in 2019 at Politecnico di Milano, with a thesis focused on the design of polymeric and hybrid materials for application in solar energy conversion. Currently she is a research fellow at Politecnico di Milano and is mainly working on the development and characterization of polymeric smart materials and of light-harvesting materials for spectral conversion in nextgeneration photovoltaics. Recently, she has expanded her research interests to include the synthesis and characterization of biodegradable and bio-derived polymers for energy storage.
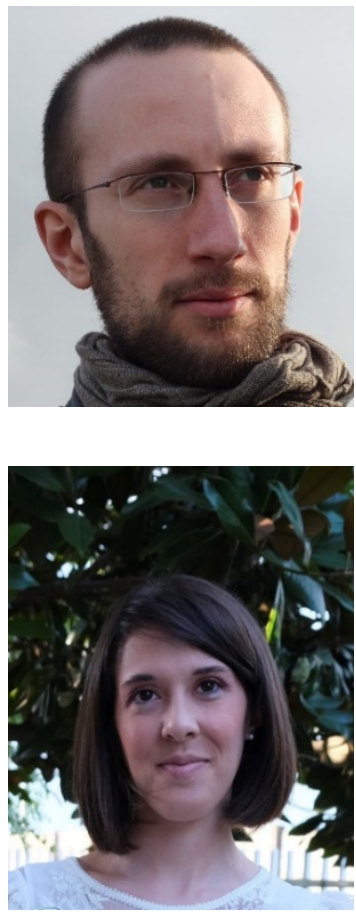


\section{References}

[1] J.W. Arnulf, Snapshot of Photovoltaics-February 2019, Energies. 12 (2019) 769. https://doi.org/10.3390/en12050769.

[2] W.G.J.H.M. Van Sark, Luminescent solar concentrators - A low cost photovoltaics alternative, Renew. Energy. 49 (2013) 207210. https://doi.org/10.1016/j.renene.2012.01.030.

[3] F. Meinardi, F. Bruni, S. Brovelli, Luminescent solar concentrators for building-integrated photovoltaics, Nat. Rev. Mater. 2 (2017) 1-9. https://doi.org/10.1038/natrevmats.2017.72.

[4] A. Goetzberger, W. Greube, Solar energy conversion with fluorescent collectors, Appl. Phys. 14 (1977) $123-139$. https://doi.org/10.1007/BF00883080.

[5] M.K. Assadi, H. Hanaei, N.M. Mohamed, R. Saidur, S. Bakhoda, R. Bashiri, M. Moayedfar, Enhancing the efficiency of luminescent solar concentrators (LSCs), Appl. Phys. A Mater. Sci. Process. 122 (2016) 1-12. https://doi.org/10.1007/s00339016-0359-2.

[6] M. Rafiee, S. Chandra, H. Ahmed, S.J. McCormack, An overview of various configurations of Luminescent Solar Concentrators for photovoltaic applications, Opt. Mater. (Amst). 91 (2019) 212-227. https://doi.org/10.1016/j.optmat.2019.01.007.

[7] B. McKenna, R.C. Evans, Towards Efficient Spectral Converters through Materials Design for Luminescent Solar Devices, Adv. Mater. 29 (2017) 1606491. https://doi.org/10.1002/adma.201606491.

[8] J.L. Banal, B. Zhang, D.J. Jones, K.P. Ghiggino, W.W.H. Wong, Emissive molecular aggregates and energy migration in luminescent solar concentrators, Acc. Chem. Res. 50 (2017) 49-57. https://doi.org/10.1021/acs.accounts.6b00432.

[9] M. Sharma, K. Gungor, A. Yeltik, M. Olutas, B. Guzelturk, Y. Kelestemur, T. Erdem, S. Delikanli, J.R. McBride, H.V. Demir, Near-Unity Emitting Copper-Doped Colloidal Semiconductor Quantum Wells for Luminescent Solar Concentrators, Adv. Mater. 29 (2017) 1-10. https://doi.org/10.1002/adma.201700821.

[10] F. Meinardi, Q.A. Akkerman, F. Bruni, S. Park, M. Mauri, Z. Dang, L. Manna, S. Brovelli, Doped Halide Perovskite Nanocrystals for Reabsorption-Free Luminescent Solar Concentrators, ACS Energy Lett. 2 (2017) 2368-2377. https://doi.org/10.1021/acsenergylett.7b00701.

[11] Y. Li, X. Zhang, Y. Zhang, R. Dong, C.K. Luscombe, Review on the Role of Polymers in Luminescent Solar Concentrators, J. Polym. Sci. Part A Polym. Chem. 57 (2019) 201-215. https://doi.org/10.1002/pola.29192.

[12] G. Griffini, Host Matrix Materials for Luminescent Solar Concentrators : Recent Achievements and Forthcoming Challenges, Front. Mater. 6 (2019) 1-8. https://doi.org/10.3389/fmats.2019.00029.

[13] G. Griffini, M. Levi, S. Turri, Novel high-durability luminescent solar concentrators based on fluoropolymer coatings, Prog. Org. Coatings. 77 (2014) 528-536. https://doi.org/10.1016/j.porgcoat.2013.11.016.

[14] G. Griffini, M. Levi, S. Turri, Novel crosslinked host matrices based on fluorinated polymers for long-term durability in thinfilm luminescent solar concentrators, Sol. Energy Mater. Sol. Cells. 118 (2013) 36-42. https://doi.org/10.1016/j.solmat.2013.05.041.

[15] E. Tatsi, G. Fortunato, B. Rigatelli, G. Lyu, S. Turri, R.C. Evans, G. Griffini, Thermoresponsive Host Polymer Matrix for SelfHealing Luminescent Solar Concentrators, ACS Appl. Energy Mater. 3 (2020) 1152-1160. https://doi.org/10.1021/acsaem.9b02196.

[16] D. Pintossi, A. Colombo, M. Levi, C. Dragonetti, S. Turri, G. Griffini, UV-curable fluoropolymers crosslinked with functional fluorescent dyes: the way to multifunctional thin-film luminescent solar concentrators, J. Mater. Chem. A. 5 (2017) 9067-9075. https://doi.org/10.1039/c7ta01692a.

[17] A. Kaniyoor, B. Mckenna, S. Comby, R.C. Evans, Design and Response of High-Efficiency, Planar, Doped Luminescent Solar Concentrators Using Organic-Inorganic Di-Ureasil Waveguides, Adv. Opt. Mater. 4 (2016) 444-456. https://doi.org/10.1002/adom.201500412.

[18] M. Sottile, G. Tomei, S. Borsacchi, F. Martini, M. Geppi, G. Ruggeri, A. Pucci, Epoxy resin doped with Coumarin 6: Example of accessible luminescent collectors, Eur. Polym. J. 89 (2017) 23-33. https://doi.org/10.1016/j.eurpolymj.2017.02.003.

[19] F. Gianfaldoni, F. De Nisi, G. Iasilli, A. Panniello, E. Fanizza, M. Striccoli, D. Ryuse, M. Shimizu, T. Biver, A. Pucci, A pushpull silafluorene fluorophore for highly efficient luminescent solar concentrators, RSC Adv. 7 (2017) 37302-37309. https://doi.org/10.1039/c7ra08022k.

[20] Y. Zhou, D. Benetti, X. Tong, L. Jin, Z.M. Wang, D. Ma, H. Zhao, F. Rosei, Colloidal carbon dots based highly stable luminescent solar concentrators, Nano Energy. 44 (2018) 378-387. https://doi.org/10.1016/j.nanoen.2017.12.017.

[21] W. Ma, W. Li, M. Cao, R. Liu, X. Zhao, X. Gong, Large Stokes-shift AIE fluorescent materials for high-performance luminescent solar concentrators, Org. Electron. 73 (2019) 226-230. https://doi.org/10.1016/j.orgel.2019.06.017.

[22] Y. Zhou, D. Benetti, Z. Fan, H. Zhao, D. Ma, A.O. Govorov, A. Vomiero, F. Rosei, Near Infrared, Highly Efficient Luminescent Solar Concentrators, Adv. Energy Mater. 6 (2016) 1-8. https://doi.org/10.1002/aenm.201501913.

[23] S. El-Molla, A.F. Mansour, A.E. Hammad, Enhancement of Fluorescence and Photostability Based on Interaction of Fluorescent Dyes with Silver Nanoparticles for Luminescent Solar Concentrators, J. Nanomater. 2017 (2017). https://doi.org/10.1155/2017/9701251.

[24] S.P. Ying, B.M. Chen, W.L. Tseng, Thin-Film Luminescent Solar Concentrators Using Inorganic Phosphors, IEEE Trans. Electron Devices. 66 (2019) 2290-2294. https://doi.org/10.1109/TED.2019.2908683.

[25] Z. Krumer, S.J. Pera, R.J.A. Van Dijk-Moes, Y. Zhao, A.F.P. De Brouwer, E. Groeneveld, W.G.J.H.M. Van Sark, R.E.I. 
Schropp, C. De Mello Donegá, Tackling self-absorption in luminescent solar concentrators with type-II colloidal quantum dots, Sol. Energy Mater. Sol. Cells. 111 (2013) 57-65. https://doi.org/10.1016/j.solmat.2012.12.028.

[26] F. Purcell-Milton, Y.K. Gun'ko, Quantum dots for Luminescent Solar Concentrators, J. Mater. Chem. 22 (2012) 16687-16697. https://doi.org/10.1039/c2jm32366d.

[27] L.R. Bradshaw, K.E. Knowles, S. McDowall, D.R. Gamelin, Nanocrystals for luminescent solar concentrators, Nano Lett. 15 (2015) 1315-1323. https://doi.org/10.1021/nl504510t.

[28] R. Mazzaro, A. Vomiero, The Renaissance of Luminescent Solar Concentrators: The Role of Inorganic Nanomaterials, Adv. Energy Mater. 8 (2018) 1-19. https://doi.org/10.1002/aenm.201801903.

[29] J. Bomm, A. Büchtemann, A.J. Chatten, R. Bose, D.J. Farrell, N.L.A. Chan, Y. Xiao, L.H. Slooff, T. Meyer, A. Meyer, W.G.J.H.M. Van Sark, R. Koole, Fabrication and full characterization of state-of-the-art quantum dot luminescent solar concentrators, Sol. Energy Mater. Sol. Cells. 95 (2011) 2087-2094. https://doi.org/10.1016/j.solmat.2011.02.027.

[30] A. Dubois, M. Canva, A. Brun, F. Chaput, J.P. Boilot, Photostability of dye molecules trapped in solid matrices, Appl. Opt. 35 (1996) 3193-3199. https://doi.org/10.1016/S0379-6779(97)80036-7.

[31] G. Griffini, L. Brambilla, M. Levi, M. Del Zoppo, S. Turri, Photo-degradation of a perylene-based organic luminescent solar concentrator: Molecular aspects and device implications, Sol. Energy Mater. Sol. Cells. 111 (2013) 41-48. https://doi.org/10.1016/j.solmat.2012.12.021.

[32] M.G. Debije, P.P.C. Verbunt, Thirty years of luminescent solar concentrator research: Solar energy for the built environment, Adv. Energy Mater. 2 (2012) 12-35. https://doi.org/10.1002/aenm.201100554.

[33] R. Riccò, S. Nizzero, E. Penna, A. Meneghello, E. Cretaio, F. Enrichi, Ultra-small dye-doped silica nanoparticles via modified sol-gel technique, J. Nanoparticle Res. 20 (2018) 1-9. https://doi.org/10.1007/s11051-018-4227-1.

[34] E. Alsolmy, W.M. Abdelwahab, A Comparative Study of Fluorescein Isothiocyanate-Encapsulated Silica Nanoparticles Prepared in Seven Different Routes for Developing Fingerprints on Non-Porous Surfaces, J. Fluoresc. 28 (2018) 1049-1058. https://doi.org/10.1007/s10895-018-2268-6.

[35] R. Herrmann, M. Rennhak, A. Reller, Synthesis and characterization of fluorescence-labelled silica core-shell and noble metaldecorated ceria nanoparticles, Beilstein J. Nanotechnol. 5 (2014) 2413-2423. https://doi.org/10.3762/bjnano.5.251.

[36] R. Nooney, C. O’Connell, S. Roy, K. Boland, G. Keegan, S. Kelleher, S. Daniels, C. McDonagh, Synthesis and characterisation of far-red fluorescent cyanine dye doped silica nanoparticles using a modified microemulsion method for application in bioassays, Sensors Actuators, B Chem. 221 (2015) 420-479. https://doi.org/10.1016/j.snb.2015.06.117.

[37] Y. Wu, L. Jiao, F. Song, M. Chen, D. Liu, W. Yang, Y. Sun, G. Hong, L. Liu, X. Peng, Achieving long-lived thermally activated delayed fluorescence in the atmospheric aqueous environment by nano-encapsulation, Chem. Commun. 55 (2019) 14522-14525. https://doi.org/10.1039/c9cc07704a.

[38] A. Burns, H. Ow, U. Wiesner, Fluorescent core-shell silica nanoparticles: Towards "lab on a particle” architectures for nanobiotechnology, Chem. Soc. Rev. 35 (2006) 1028-1042. https://doi.org/10.1039/b600562b.

[39] B. Cohen, C. Martin, S.K. Iyer, U. Wiesner, A. Douhal, Single Dye Molecule Behavior in Fluorescent Core - Shell Silica Nanoparticles, Chem. Mater. 24 (2012) 361-372. https://doi.org/10.1021/cm203196w.

[40] X. Mu, C.L. Wu, J.P. Lai, J. Bin Chen, J.S. Zheng, C. Li, Y.B. Zhao, A facile and general approach for the synthesis of fluorescent silica nanoparticles doped with inert dyes, Chinese Sci. Bull. 56 (2011) 3242-3246. https://doi.org/10.1007/s11434011-4727-1.

[41] R. Ciriminna, M. Sciortino, G. Alonzo, A. De Schrijver, M. Pagliaro, From Molecules to Systems : Sol - Gel Microencapsulation in Silica-Based Materials, Chem. Soc. Rev. 111 (2011) 765-789.

[42] X. Zhao, R.P. Bagwe, W. Tan, Development of organic-dye-doped silica nanoparticles in a reverse microemulsion, Adv. Mater. 16 (2004) 173-176. https://doi.org/10.1002/adma.200305622.

[43] R.P. Bagwe, C. Yang, L.R. Hilliard, W. Tan, Optimization of dye-doped silica nanoparticles prepared using a reverse microemulsion method, Langmuir. 20 (2004) 8336-8342. https://doi.org/10.1021/la049137j.

[44] C. Xie, D. Yin, J. Li, B. Liu, M. Wu, Preparation of Luminescent Dye Doped Core-Shell Nanoparticles and Their Application in cell recognition, in: 2010 3rd Int. Nanoelectron. Conf. (INEC); IEEE, 2010: pp. 1005-1006.

[45] W. Stöber, A. Fink, E. Bohn, Controlled growth of monodisperse silica spheres in the micron size range, J. Colloid Interface Sci. 26 (1968) 62-69. https://doi.org/10.1016/0021-9797(68)90272-5.

[46] R. Tapec, X.J. Zhao, W. Tan, Development of Organic Dye-Doped Silica Nanoparticles for Bioanalysis and Biosensors, J. Nanosci. Nanotechnol. 2 (2002) 405-409. https://doi.org/10.1166/jnn.2002.114.

[47] C. Li, H. Wonneberger, Perylene imides for organic photovoltaics: Yesterday, today, and tomorrow, Adv. Mater. 24 (2012) 613-636. https://doi.org/10.1002/adma.201104447.

[48] A. Nowak-Król, F. Würthner, Progress in the synthesis of perylene bisimide dyes, Org. Chem. Front. 6 (2019) 1272-1318. https://doi.org/10.1039/c8qo01368c.

[49] A.S. Ethiraj, N. Hebalkar, S. Kharrazi, J. Urban, S.R. Sainkar, S.K. Kulkarni, Photoluminescent core-shell particles of organic dye in silica, J. Lumin. 114 (2005) 15-23. https://doi.org/10.1016/j.jlumin.2004.11.005.

[50] A.I. Journal, Y. Hu, J. Chen, Z. Zhang, N. Zhang, D. Li, Preparation, characterization of Rhodamine 6G nanocomposites and their application in labelling cancer cells, Integr. Ferroelectr. 169 (2016) 140-145. https://doi.org/10.1080/10584587.2016.1165565.

[51] L. Wang, K. Wang, S. Santra, X. Zhao, L.R. Hilliard, J.E. Smith, Y. Wu, W. Tan, Watching silica nanoparticles glow in the biological world, Anal. Chem. 78 (2006) 646-654. https://doi.org/10.1021/ac0693619. 
[52] E. Alsolmy, W.M. Abdelwahab, G. Patonay, A Comparative Study of Fluorescein Isothiocyanate-Encapsulated Silica Nanoparticles Prepared in Seven Different Routes for Developing Fingerprints on Non-Porous Surfaces, J. Fluoresc. 28 (2018) 1049-1058. https://doi.org/10.1007/s10895-018-2268-6.

[53] A.S. Ethiraj, S. Kharrazi, N. Hebalkar, J. Urban, S.R. Sainkar, S.K. Kulkarni, Highly photostable dye entrapped core-shell particles, Mater. Lett. 61 (2007) 4738-4742. https://doi.org/10.1016/j.matlet.2007.03.030.

[54] B.J. Theaker, K.E. Hudson, F.J. Rowell, Doped hydrophobic silica nano- and micro-particles as novel agents for developing latent fingerprints, Forensic Sci. Int. 174 (2008) 26-34. https://doi.org/10.1016/j.forsciint.2007.02.030.

[55] F.F.E. Kohle, J.A. Hinckley, U.B. Wiesner, Dye Encapsulation in Fluorescent Core-Shell Silica Nanoparticles as Probed by Fluorescence Correlation Spectroscopy, J. Phys. Chem. C Nanomater. Interfaces. 123 (2019) 9813-9823. https://doi.org/10.1016/j.physbeh.2017.03.040.

[56] D. Sriramulu, S.P. Turaga, A.A. Bettiol, S. Valiyaveettil, Molecular Organization Induced Anisotropic Properties of Perylene Silica Hybrid Nanoparticles, Sci. Rep. 7 (2017) 1-11. https://doi.org/10.1038/s41598-017-07892-4.

[57] H.S. Muddana, T.T. Morgan, J.H. Adair, P.J. Butler, Photophysics of Cy3-encapsulated calcium phosphate nanoparticles, Nano Lett. 9 (2009) 1559-1566. https://doi.org/10.1021/nl803658w.

[58] D.R. Larson, H. Ow, H.D. Vishwasrao, A.A. Heikal, U. Wiesner, W.W. Webb, Silica nanoparticle architecture determines radiative properties of encapsulated fluorophores, Chem. Mater. 20 (2008) 2677-2684. https://doi.org/10.1021/cm7026866.

[59] X. Sun, Y. Xu, D. Jiang, D. Yang, D. Wu, Y. Sun, Y. Yang, H. Yuan, F. Deng, Study on the ammonia-catalyzed hydrolysis kinetics of single phenyltriethoxysilane and mixed phenyltriethoxysilane/tetraethoxysilane systems by liquid-state 29Si NMR, Colloids Surfaces A Physicochem. Eng. Asp. 289 (2006) 149-157. https://doi.org/10.1016/j.colsurfa.2006.04.024.

[60] U.S. Schubert, N. Hüsing, Synthesis of Inorganic Materials, John Wiley \& Sons, London, UK, 2019.

[61] F. Würthner, T.E. Kaiser, C.R. Saha-Möller, J-aggregates: From serendipitous discovery to supramolecular engineering of functional dye materials, Angew. Chemie - Int. Ed. 50 (2011) 3376-3410. https://doi.org/10.1002/anie.201002307.

[62] G. Griffini, M. Levi, S. Turri, Thin-film luminescent solar concentrators: A device study towards rational design, Renew. Energy. 78 (2015) 288-294. https://doi.org/10.1016/j.renene.2015.01.009.

[63] H. Yoo, J. Yang, A. Yousef, M.R. Wasielewski, D. Kim, Excimer formation dynamics of intramolecular $\pi$-stacked perylenediimides probed by single-molecule fluorescence spectroscopy, J. Am. Chem. Soc. 132 (2010) 3939-3944. https://doi.org/10.1021/ja910724x.

[64] C. Haines, M. Chen, K.P. Ghiggino, The effect of perylene diimide aggregation on the light collection efficiency of luminescent concentrators, Sol. Energy Mater. Sol. Cells. 105 (2012) 287-292. https://doi.org/10.1016/j.solmat.2012.06.030.

[65] A. Pedone, G. Prampolini, S. Monti, V. Barone, Realistic modeling of fluorescent dye-doped silica nanoparticles: A step toward the understanding of their enhanced photophysical properties, Chem. Mater. 23 (2011) 5016-5023. https://doi.org/10.1021/cm202436b.

[66] Q. Zheng, M.F. Juette, S. Jockusch, M.R. Wasserman, Z. Zhou, R.B. Altman, S.C. Blanchard, Ultra-stable organic fluorophores for single-molecule research, Chem. Soc. Rev. 43 (2014) 1044-1056. https://doi.org/10.1039/c3cs60237k.

[67] I. Baumberg, O. Berezin, A. Drabkin, B. Gorelik, L. Kogan, M. Voskobojnik, M. Zaidman, Effect of polymer matrix on photostability of photo-luminescent dyes in multi-layer polymeric structures, Polym. Degrad. Stab. 73 (2001) $403-410$. https://doi.org/10.1016/S0141-3910(01)00119-7.

[68] N. Steinbrück, M. Könemann, G. Kickelbick, Effect of polysiloxane encapsulation material compositions on emission behaviour and stabilities of perylene dyes, RSC Adv. 8 (2018) 18128-18138. https://doi.org/10.1039/c8ra01700j.

[69] D. Cambié, F. Zhao, V. Hessel, M.G. Debije, T. Noël, A Leaf-Inspired Luminescent Solar Concentrator for Energy-Efficient Continuous-Flow Photochemistry, Angew. Chemie - Int. Ed. 56 (2017) 1050-1054. https://doi.org/10.1002/anie.201611101.

[70] C. Tummeltshammer, A. Taylor, A.J. Kenyon, I. Papakonstantinou, Flexible and fluorophore-doped luminescent solar concentrators based on polydimethylsiloxane, Opt. Lett. 41 (2016) 713. https://doi.org/10.1364/ol.41.000713.

[71] I.A. Carbone, K.R. Frawley, M.K. McCann, Flexible, Front-Facing Luminescent Solar Concentrators Fabricated from Lumogen F Red 305 and Polydimethylsiloxane, Int. J. Photoenergy. 2019 (2019) 1-9. https://doi.org/10.1155/2019/8680931.

[72] M. Buffa, S. Carturan, M.G. Debije, A. Quaranta, G. Maggioni, Dye-doped polysiloxane rubbers for luminescent solar concentrator systems, Sol. Energy Mater. Sol. Cells. 103 (2012) 114-118. https://doi.org/10.1016/j.solmat.2012.04.019.

[73] C. Tummeltshammer, M. Portnoi, S.A. Mitchell, A.T. Lee, A.J. Kenyon, A.B. Tabor, I. Papakonstantinou, On the ability of Förster resonance energy transfer to enhance luminescent solar concentrator efficiency, Nano Energy. 32 (2017) 263-270. https://doi.org/10.1016/j.nanoen.2016.11.058.

[74] G. Lyu, J. Kendall, I. Meazzini, E. Preis, S. Bayseç, U. Scherf, S. Clément, R.C. Evans, Luminescent Solar Concentrators Based on Energy Transfer from an Aggregation-Induced Emitter Conjugated Polymer, ACS Appl. Polym. Mater. 1 (2019) 3039-3047. https://doi.org/10.1021/acsapm.9b00718.

[75] J. ter Schiphorst, M.L.M.K.H.Y.K. Cheng, M. van der Heijden, R.L. Hageman, E.L. Bugg, T.J.L. Wagenaar, M.G. Debije, Printed luminescent solar concentrators: Artistic renewable energy, Energy Build. 207 (2020) 27-30. https://doi.org/10.1016/j.enbuild.2019.109625.

[76] L.J. Brennan, F. Purcell-Milton, B. McKenna, T.M. Watson, Y.K. Gun’ko, R.C. Evans, Large area quantum dot luminescent solar concentrators for use with dye-sensitised solar cells, J. Mater. Chem. A. 6 (2018) 2671-2680. https://doi.org/10.1039/c7ta04731b.

[77] Y. Zhou, H. Zhao, D. Ma, F. Rosei, Harnessing the properties of colloidal quantum dots in luminescent solar concentrators, Chem. Soc. Rev. 47 (2018) 5866-5890. https://doi.org/10.1039/c7cs00701a. 
[78] G. Liu, R. Mazzaro, Y. Wang, H. Zhao, A. Vomiero, High efficiency sandwich structure luminescent solar concentrators based on colloidal quantum dots, Nano Energy. 60 (2019) 119-126. https://doi.org/10.1016/j.nanoen.2019.03.038.

[79] L. Zdražil, S. Kalytchuk, K. Holá, M. Petr, O. Zmeškal, Š. Kment, A.L. Rogach, R. Zbořil, A carbon dot-based tandem luminescent solar concentrator, Nanoscale. 12 (2020) 6664-6672. https://doi.org/10.1039/c9nr10029f.

[80] P. Moraitis, R.E.I. Schropp, W.G.J.H.. Van Sark, Nanoparticles for Luminescent Solar Concentrators - A review, Opt. Mater. (Amst). 84 (2018) 636-645. https://doi.org/10.1016/j.optmat.2018.07.034.

[81] Y. You, X. Tong, W. Wang, J. Sun, P. Yu, H. Ji, X. Niu, Z.M. Wang, Eco-Friendly Colloidal Quantum Dot-Based Luminescent Solar Concentrators, Adv. Sci. 6 (2019). https://doi.org/10.1002/advs.201801967.

[82] A. Vomiero, X. Gong, S. You, H. Zhao, G. Liu, X. Wang, C. Sun, B. Liu, Y. Zhang, G. Han, M. Zavelani-Rossi, F.V.A. Camargo, Gram-Scale Synthesis of Carbon Quantum Dots with Large Stokes Shift Enables Fabrication of Eco-Friendly and High-Efficiency Luminescent Solar Concentrators, Energy Environ. Sci. (2020). https://doi.org/10.1039/D0EE02235G.

[83] J.L. Banal, H. Soleimaninejad, F.M. Jradi, M. Liu, J.M. White, A.W. Blakers, M.W. Cooper, D.J. Jones, K.P. Ghiggino, S.R. Marder, T.A. Smith, W.W.H. Wong, Energy Migration in Organic Solar Concentrators with a Molecularly Insulated Perylene Diimide, J. Phys. Chem. C. 120 (2016) 12952-12958. https://doi.org/10.1021/acs.jpcc.6b04479.

[84] C. Yang, D. Liu, R.R. Lunt, How to Accurately Report Transparent Luminescent Solar Concentrators, Joule. 3 (2019) 28712876. https://doi.org/10.1016/j.joule.2019.10.009.

[85] J.R. Schrecengost, S.D. Bowser, S.W. Weible, J.M. Solomon, L.J. Minner, J.T. Gresh, B.P. Wittmershaus, Increasing the area of a white scattering background can increase the power output of a luminescent solar concentrator, Sol. Energy. 170 (2018) 132-137. https://doi.org/10.1016/j.solener.2018.05.022.

[86] M.G. Debije, J. Teunissen, M.J. Kastelijn, P.P.C. Verbunt, W.M. Bastiaansen, The effect of a scattering layer on the edge output of a luminescent solar concentrator, Sol. Energy Mater. Sol. Cells. 93 (2009) 1345-1350. https://doi.org/10.1016/j.solmat.2009.02.013.

[87] H. Zhao, G. Liu, G. Han, High-performance laminated luminescent solar concentrators based on colloidal carbon quantum dots, Nanoscale Adv. 1 (2019) 4888-4894. https://doi.org/10.1039/c9na00527g. 


\section{Figures}
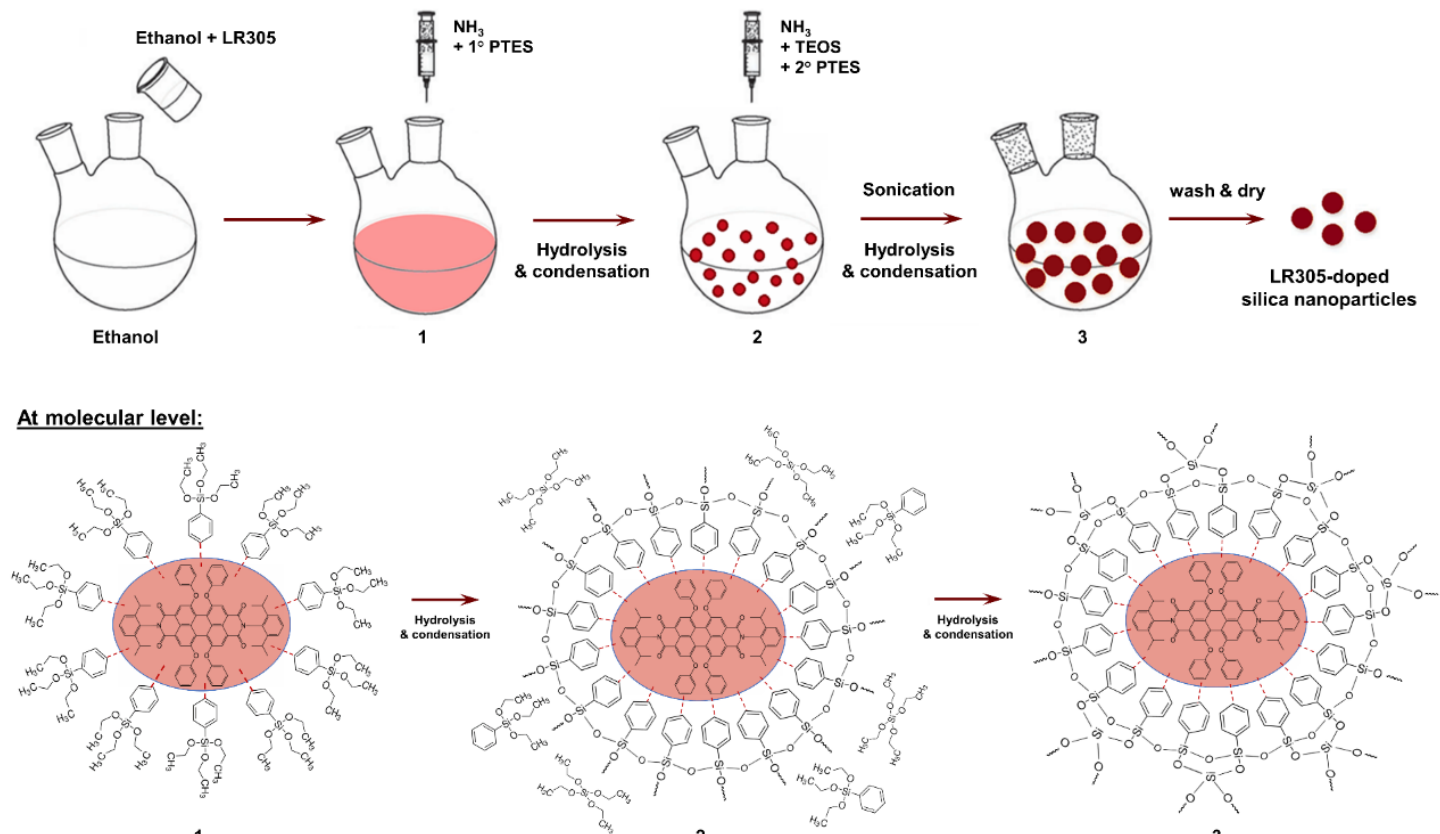

Figure 1. Top: schematic diagram of the synthetic procedure to prepare SiLRNPs. Bottom: molecular representation of the two-step process to obtain SiLRNPs, with initial formation of a layer of PTES around LR305 molecules and then formation of the silica shell upon condensation of PTES and TEOS. 


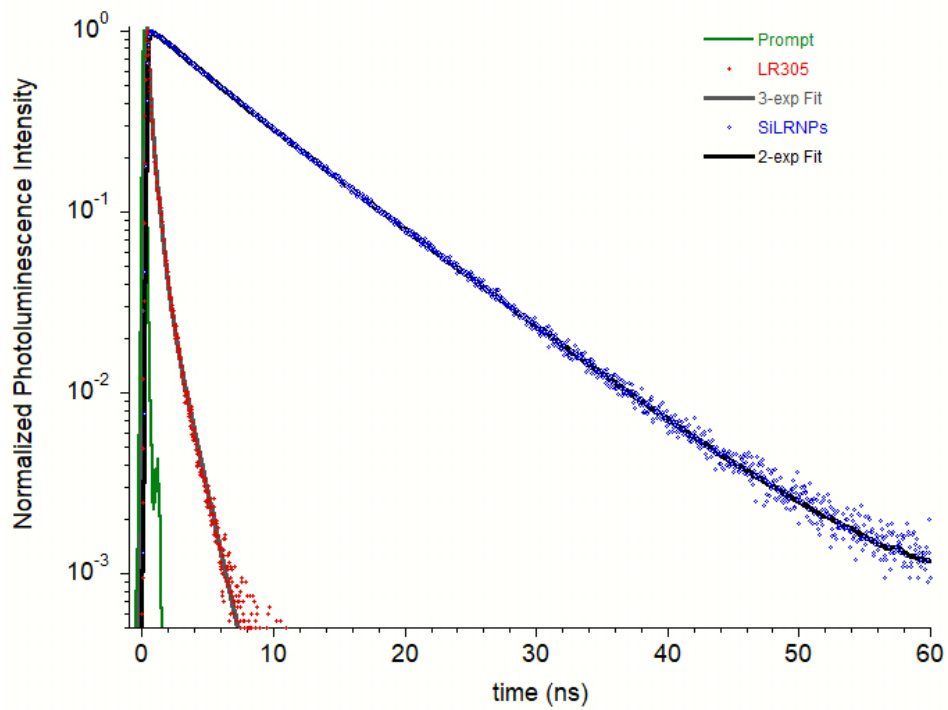

Figure 2. Time-resolved fluorescence decays of LR305 and SiLRNPs in solid state. Excitation 408nm, emission at 656nm. 


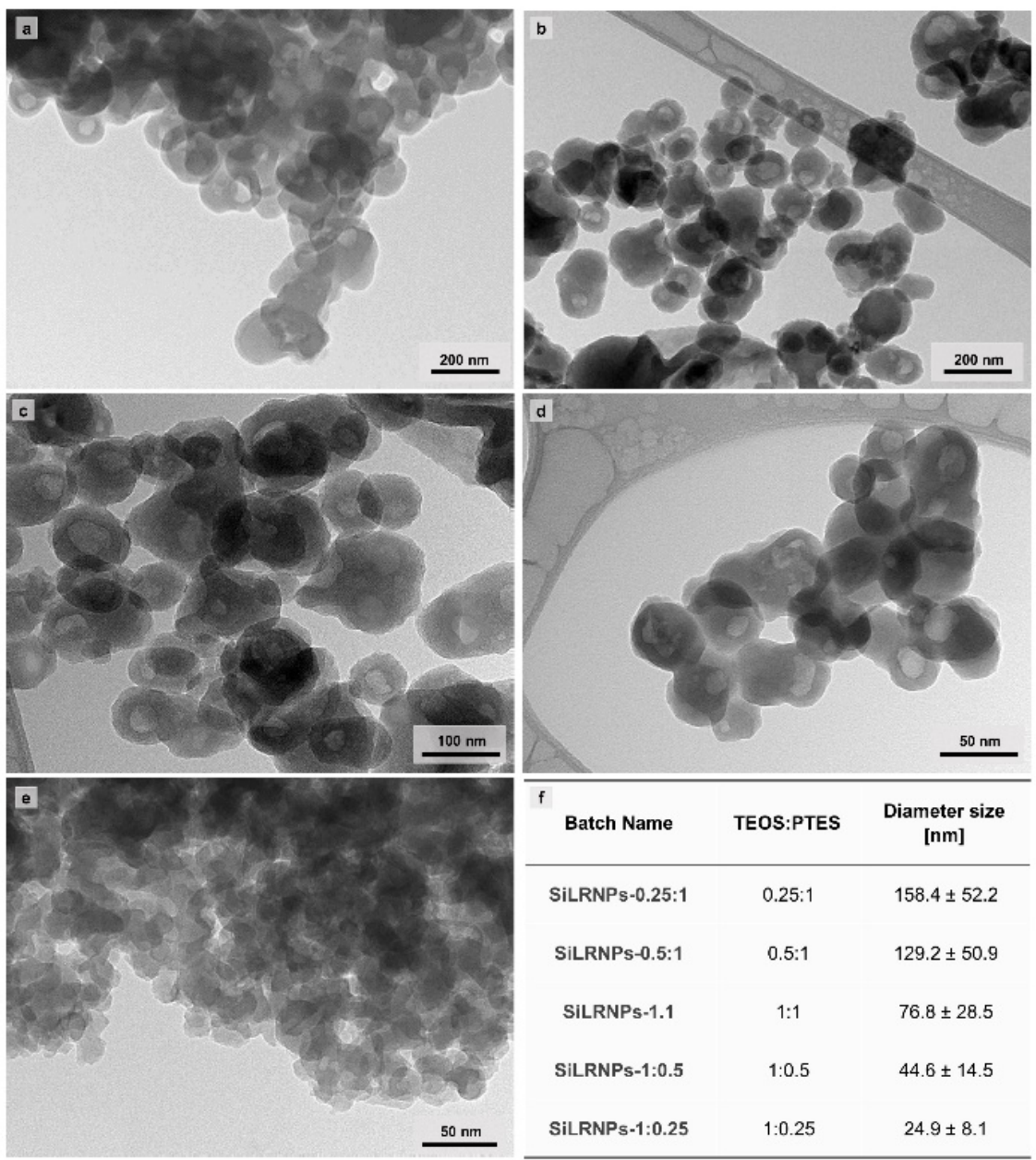

Figure 3. TEM micrographs of SiLRNPs prepared with TEOS:PTES volume ratio (a) 0.25:1 (b) 0.5:1 (c) 1:1 (d) 1:0.5 (e) 1:0.25. (f) Table reporting the values of average NP size for all formulations, as inferred from TEM and SEM analysis. 

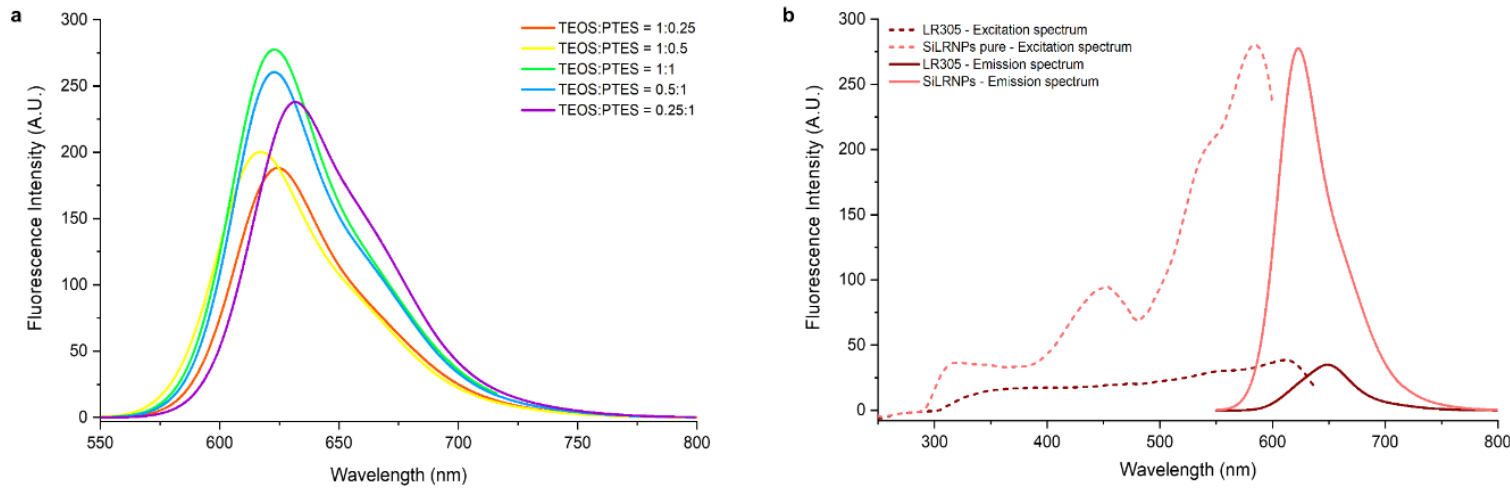

Figure 4. (a) Emission spectra of $1 \mathrm{mg}$ of solid powdered SiLRNPs with TEOS:PTES = 1:0.25, TEOS:PTES = 1:0.5, TEOS:PTES = 1:1, TEOS:PTES $=0.5: 1$, TEOS:PTES $=0.25: 1$. (b) Excitation and emission spectra of $1 \mathrm{mg}$ of LR305 and SiLRNPs-1:1 in solid powder form.

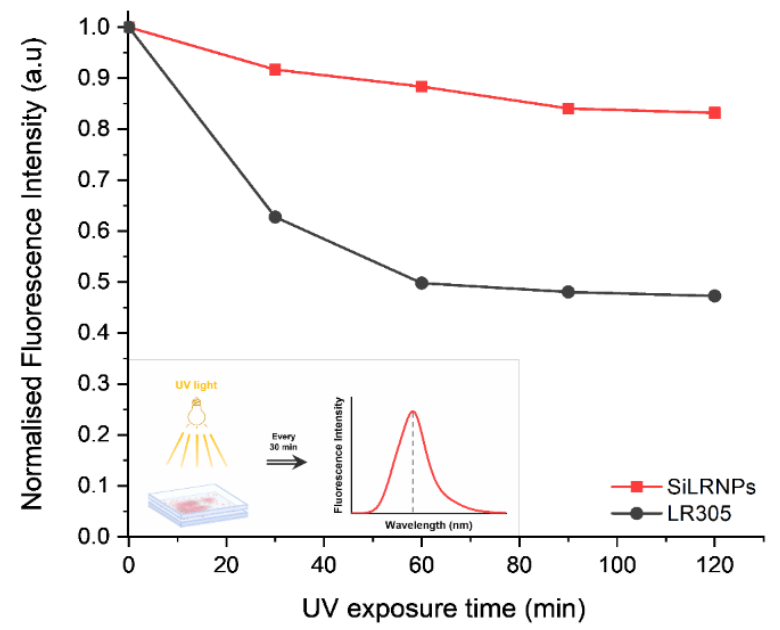

Figure 5. Normalized fluorescence intensity vs. UV exposure time of pure LR305 and SiLRNPs. In the inset: experimental setup for accelerated aging measurements, carried out with fluorescence spectroscopy during exposure to UV radiation. 

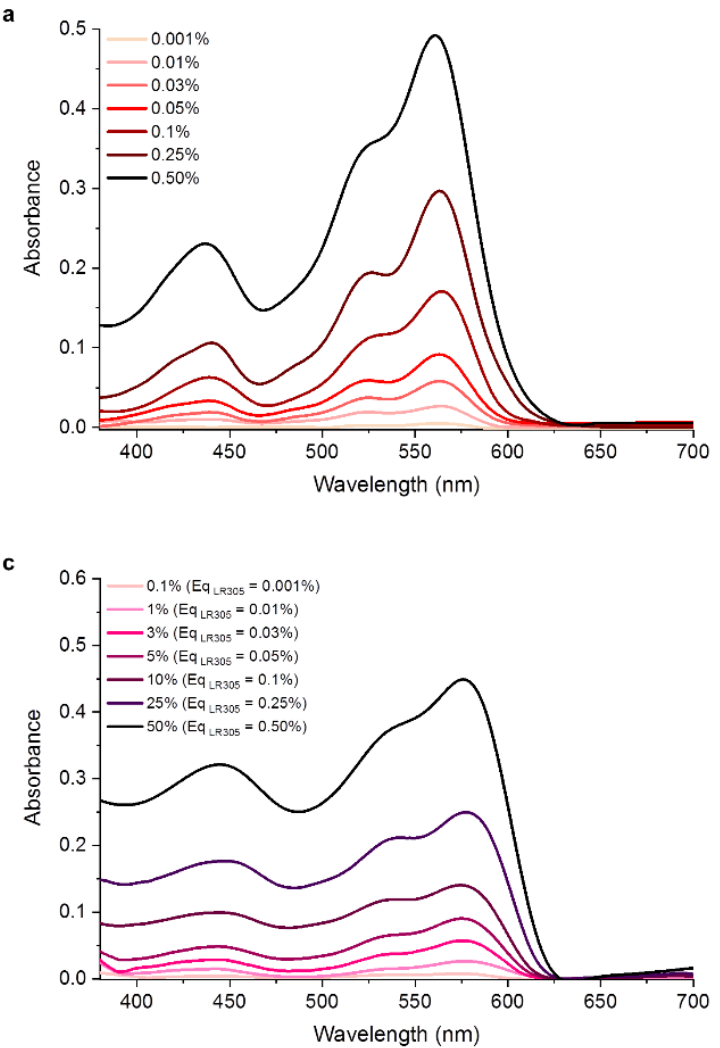

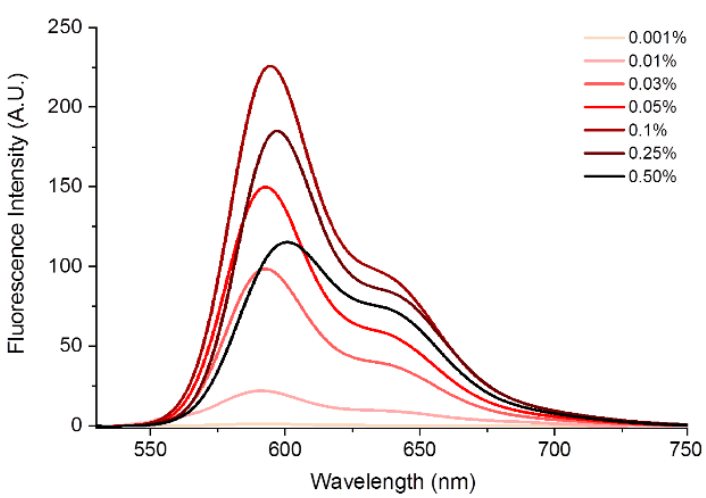

d

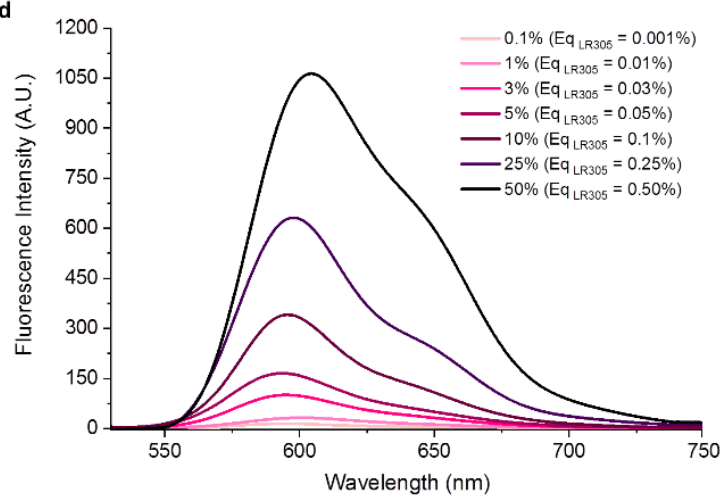

Figure 6. (a) Absorption and (b) front-face fluorescence spectra of LR305-PDMS LSC devices in thin-film configuration with increasing LR305 concentration (wt.\%). (c) Absorption and (d) front-face fluorescence spectra of SiLRNPs-PDMS LSC devices in thin-film configuration with increasing SiLRNP concentration (wt.\%). Eq $q_{\mathrm{LR} 305}$ stands for "equivalent LR305 concentration" calculated based on the fact that $\sim 1 \mathrm{wt} \%$ of LR305 is entrapped in SiLRNPs with TEOS:PTES $=0.25: 1 \mathrm{v} / \mathrm{v}$. 


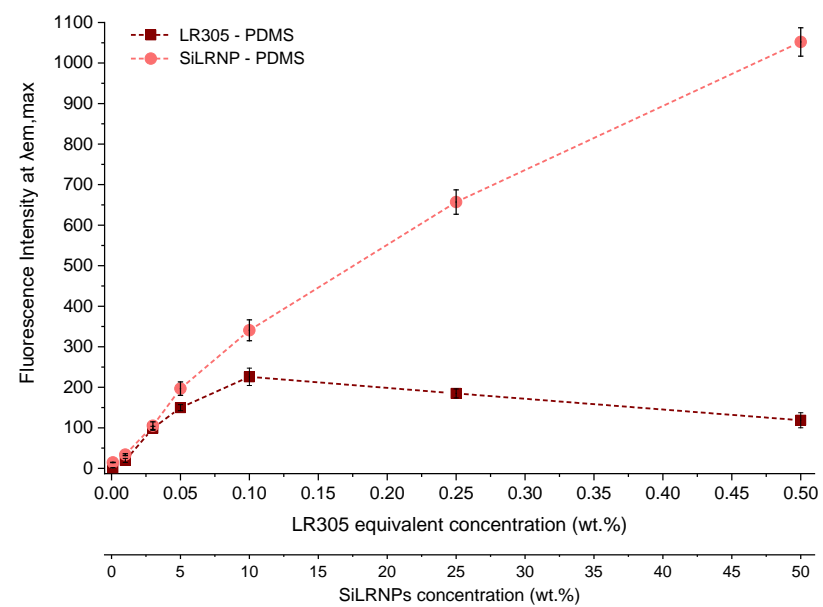

Figure 7. Maximum fluorescence intensity vs. LR305 equivalent concentration (and SiLRNPs concentration) of LR305-PDMS and SiLRNP-PDMS LSC devices in thin-film configuration.
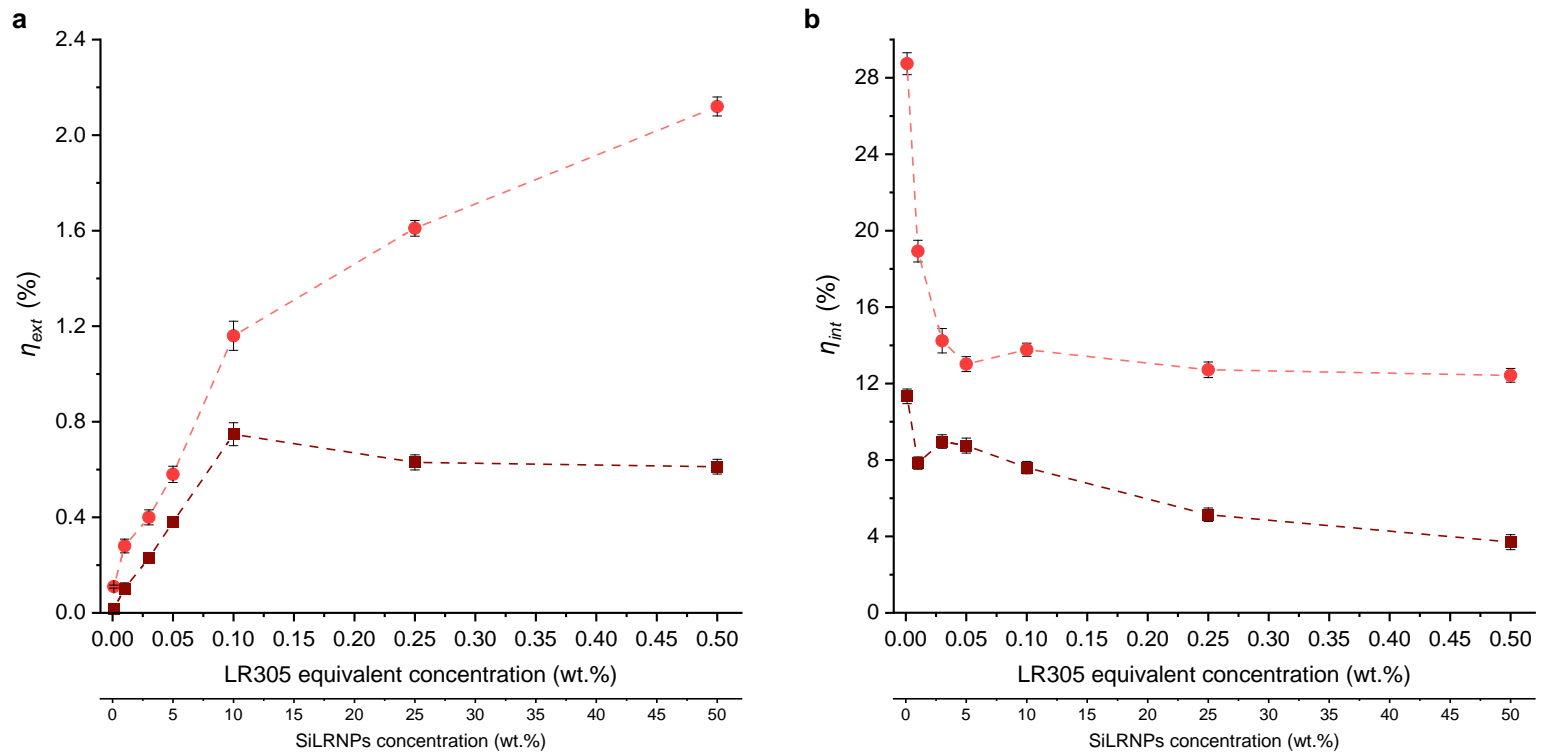

Figure 8. (a) External photon efficiency $\left(\eta_{\text {ext }}\right)$ and $(b)$ internal photon efficiency $\left(\eta_{\text {int }}\right)$ of LR305- and SiLRNPs- PDMS LSCs as a function of luminophore content (wt.\%). The values and errors reported for $\eta_{\mathrm{ext}}$ and $\eta_{\text {int }}$ are the mean and standard deviation of three repeat measurements, respectively. 
Table 1. $\eta_{d e v, \text { dark background }}$ and $\eta_{\text {dev,white scatterer }}$ calculated for PDMS-based LSCs at increasing luminophore concentration. Values in brackets indicate the amount (wt.\%) of LR305 in LR305-PDMS LSCs or SiLRNPs in SiLRNPs-PDMS LSCs.

\begin{tabular}{llll}
\hline & $\begin{array}{l}\text { LR305 equivalent } \\
\text { concentration (wt.\%) }\end{array}$ & $\eta_{\text {dev,dark background }(\%)}$ & $\eta_{\text {dev, white scatterer }(\%)}$ \\
\hline LR305-PDMS LSC (0.001 wt.) & 0.001 & $0.021 \pm 0.013$ & $0.029 \pm 0.008$ \\
LR305-PDMS LSC (0.01 wt.\%) & 0.01 & $0.060 \pm 0.005$ & $0.094 \pm 0.019$ \\
LR305-PDMS LSC (0.03 wt.\%) & 0.03 & $0.097 \pm 0.003$ & $0.141 \pm 0.002$ \\
LR305-PDMS LSC (0.05 wt.\%) & 0.05 & $0.132 \pm 0.007$ & $0.181 \pm 0.008$ \\
LR305-PDMS LSC (0.1 wt.\%) & 0.1 & $0.237 \pm 0.009$ & $0.321 \pm 0.011$ \\
LR305-PDMS LSC (0.25 wt.\%) & 0.25 & $0.199 \pm 0.005$ & $0.307 \pm 0.014$ \\
LR305-PDMS LSC (0.5 wt.\%) & 0.5 & $0.183 \pm 0.016$ & $0.289 \pm 0.009$ \\
& & & \\
SiLRNPs-PDMS LSC (0.1 wt.\%) & 0.001 & $0.041 \pm 0.010$ & $0.064 \pm 0.004$ \\
SiLRNPs-PDMS LSC (1 wt.\%) & 0.01 & $0.123 \pm 0.005$ & $0.181 \pm 0.014$ \\
SiLRNPs-PDMS LSC (3 wt.\%) & 0.03 & $0.196 \pm 0.009$ & $0.284 \pm 0.015$ \\
SiLRNPs-PDMS LSC (5 wt.\%) & 0.05 & $0.290 \pm 0.003$ & $0.321 \pm 0.012$ \\
SiLRNPs-PDMS LSC (10 wt.\%) & 0.1 & $0.489 \pm 0.004$ & $0.546 \pm 0.012$ \\
SiLRNPs-PDMS LSC (25 wt.\%) & 0.25 & $0.774 \pm 0.013$ & $0.995 \pm 0.017$ \\
SiLRNPs-PDMS LSC (50 wt.\%) & 0.5 & $1.073 \pm 0.010$ & $1.287 \pm 0.011$ \\
& & & \\
\hline
\end{tabular}

